\title{
Combined Spinal and Caudal Epidural Anesthesia for Prolonged Surgical Procedures in Pediatric-Aged Patients: A Report of Two Cases
}

\author{
Emily D. Geyer ${ }^{\mathrm{a}}$, David P. Martina, b, Tarun Bhalla ${ }^{\mathrm{a}, \mathrm{b}}$, Venkata R. Jayanthic, d, \\ Joseph D. Tobias ${ }^{\mathrm{a}, \mathrm{b}, \mathrm{e}}$, Emmett E. Whitaker ${ }^{\mathrm{a}, \mathrm{b}}$
}

\begin{abstract}
With emerging evidence that specific anesthetic agents may affect neurocognitive outcomes, there has been renewed interest in the applications of spinal anesthesia in neonates and infants. Because of its short duration of action, spinal anesthesia in infants is generally limited to procedures lasting less than $70-75 \mathrm{~min}$. To avoid this limitation, we report a technique combining spinal and caudal epidural anesthesia. We present two infants in whom this technique was used to provide surgical anesthesia (without general anesthesia or airway manipulation) for two cases lasting more than $90 \mathrm{~min}$. The technique used is presented, its applications discussed, and previous reports reviewed.
\end{abstract}

Keywords: Regional anesthesia; Pediatric; Neurotoxicity; Safety

\section{Introduction}

Spinal anesthesia (SA) became a popular technique during the 1980 s when it was used as an alternative to general anesthesia (GA) as a means of avoiding postoperative apnea in high-risk, former preterm neonates [1]. However, with the introduction of sevoflurane into clinical practice, the risk of apnea was shown to be limited and interest in SA waned [2]. More recently, there have been numerous reports in the literature regarding the potential long-term neurocognitive effects of GA during infancy and the neonatal period $[3,4]$. While there is insufficient pro-

Manuscript submitted December 21, 2017, accepted January 15, 2018

aDepartment of Anesthesiology and Pain Medicine, Nationwide Children's Hospital, Columbus, OH, USA

bepartment of Anesthesiology and Pain Medicine, The Ohio State University College of Medicine, Columbus, OH, USA

'Division of Pediatric Urology, Nationwide Children's Hospital, Columbus, $\mathrm{OH}, \mathrm{USA}$

dDepartment of Urology, The Ohio State University College of Medicine, Columbus, OH, USA

${ }^{\text {e}}$ Corresponding Author: Joseph D. Tobias, Department of Anesthesiology and Pain Medicine, Nationwide Children's Hospital, 700 Children's Drive, Columbus, OH 43205, USA. Email: Joseph.Tobias@nationwidechildrens.org

doi: https://doi.org/10.14740/jmc2994w spective evidence to clearly prove a causal relationship, there has been a renewed interest in the use of SA as a means of avoiding GA during the potentially vulnerable time period. Given its limited duration of action, single-shot SA is applicable only for surgical procedures that can be accomplished in less than 70 75 min. We present two infants in whom a combined SA with a caudal epidural catheter technique was used to provide surgical anesthesia for cases lasting more than $90 \mathrm{~min}$. The technique is presented, its applications discussed, and previous reports from the literature regarding the combined use of spinal and epidural anesthesia in the pediatric population reviewed.

\section{Case Report}

Institutional Review Board approval is not required for presentation of case series with two or fewer patients at Nationwide Children's Hospital (Columbus, $\mathrm{OH}$ ).

\section{Case 1}

At the time of the surgery, the patient was a 2 -year-old, $11.2 \mathrm{~kg}$ boy scheduled for first stage hypospadias repair. He had no allergies. His medication history was significant only for albuterol and cetirizine, which he took for wheezing (reactive airway disease) and seasonal allergies, respectively. There was also a history of previous problems with general anesthesia and an unexplained cardiac arrest during an orchidopexy. Although a thorough postprocedure cardiac evaluation was negative, the patient's parents were motivated to avoid GA for subsequent procedures. For this first stage hypospadias repair, SA alone would not be sufficient due to the length of the procedure. Informed consent was obtained for combined spinal anesthesia and caudal epidural anesthesia with sedation. The patient's vital signs on the morning of the procedure were unremarkable. In the preoperative area, LMX cream (4\% topical lidocaine) was applied to the lumbar spine to minimize discomfort with placement of the spinal needle. After arrival to the operating room, the patient was placed in the sitting position. After sterile preparation, the intrathecal space was accessed on the first attempt using a 1.5 inch, 22-gauge spinal needle. Upon return of cerebrospinal fluid, $1.2 \mathrm{~mL}$ of $0.5 \%$ bupivacaine-epinephrine 1:200,000 with clonidine $(11 \mu \mathrm{g})$ was administered. Following successful SA, the patient was then 
Table 1. Previous Reports of Regional Anesthesia for Prolonged Surgical Procedures

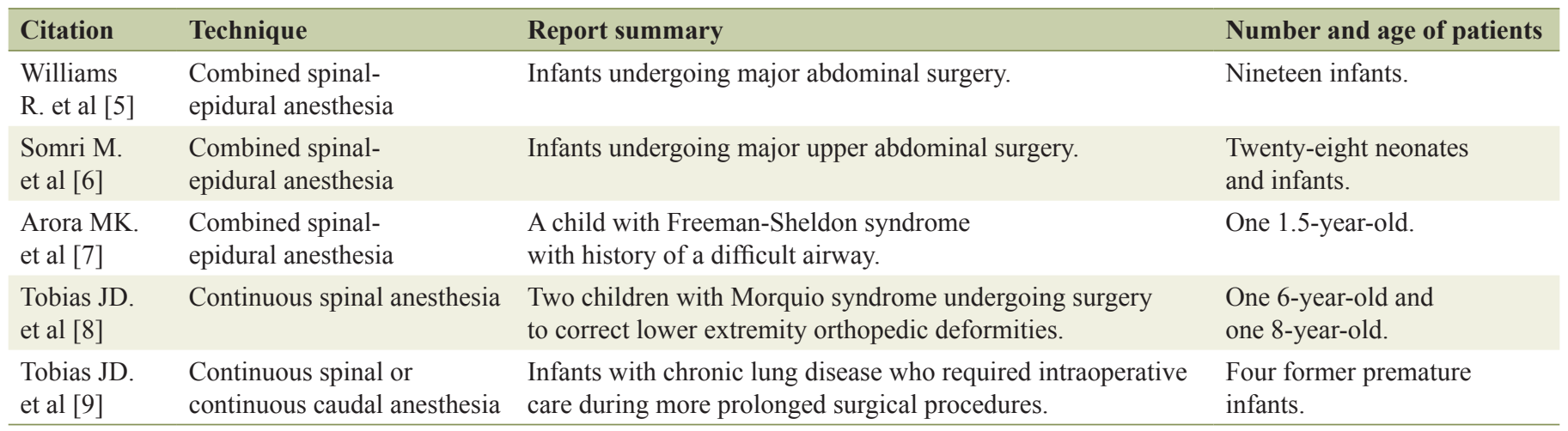

placed in the supine position, standard monitors were placed, and a 22-gauge peripheral intravenous catheter was placed in the right foot. Immediate sensory and motor block were noted. A single dose of dexmedetomidine (total of $12 \mu \mathrm{g}$ in divided doses) was administered intravenously for sedation during caudal epidural catheter placement. The patient was placed in right lateral decubitus position for caudal epidural catheter placement. After sterile preparation, the caudal epidural space was accessed using an 18-gauge Tuohy needle. A 20-gauge caudal catheter was advanced $3 \mathrm{~cm}$ into the epidural space to allow sacral coverage. The needle was removed and the catheter secured. A test dose of lidocaine $1.5 \%$ with epinephrine 1:200,000 (1 mL) was negative. The patient was placed supine and the surgical procedure started under SA. Dexmedetomidine $(0.5-1 \mu \mathrm{g} / \mathrm{kg} / \mathrm{h})$ was administered intravenously for intraoperative sedation. Approximately $1 \mathrm{~h}$ after the start of the surgical procedure, the caudal catheter was dosed with $3 \%$ chloroprocaine $(1 \mathrm{~mL} / \mathrm{kg}$ ) followed by a continuous infusion of $3 \%$ chloroprocaine $(1.5 \mathrm{~mL} / \mathrm{kg} / \mathrm{h})$. The surgery was completed with excellent operating conditions. The patient was hemodynamically stable and required no supplemental oxygen throughout the procedure, which lasted approximately $2 \mathrm{~h}$. The caudal catheter was removed in the operating room following completion of the surgery. The patient's post-anesthesia care unit (PACU) stay was uncomplicated and he was discharged home that afternoon without incident. The parents were very satisfied with the care of their son, and they requested the same approach for his second-stage hypospadias repair, which was also performed successfully.

\section{Case 2}

At the time of the surgery, the patient was a 16-month-old, $8.75 \mathrm{~kg}$ girl scheduled for bilateral ureteral reimplantation. The patient had a known history of Tetralogy of Fallot (TOF) status post repair, grade 4 primary vesicoureteral reflux, recurrent pyelonephritis, and difficult postoperative pain management. She had no allergies. Her medication history was significant only for trimethoprim-sulfamethoxazole for urinary tract infection prophylaxis. Preoperative laboratory studies were not performed and her vital signs on the morning of the procedure were unremarkable. An echocardiogram performed 2 months before the surgery revealed findings typical for a patient that had undergone TOF repair with normal biventricular function. The patient had previously received GA uneventfully, but her parents desired avoidance of GA due to her history of congenital heart disease. Informed consent was obtained for combined spinal anesthesia and caudal epidural anesthesia. In the preoperative area, LMX cream ( $4 \%$ topical lidocaine) was applied to the lumbar to minimize discomfort with the placement of the spinal needle. After arrival to the operating room, the patient was placed in the sitting position. After sterile preparation, the intrathecal space was accessed on the first attempt using a 1.5 inch, 22-gauge spinal needle. Upon return of cerebrospinal fluid, $1.2 \mathrm{~mL}$ of $0.5 \%$ bupivacaine with epinephrine $1: 200,000$ was administered. Following successful SA, the patient was placed in the supine position, standard monitors were placed, and a 22-gauge peripheral intravenous catheter was placed in the left foot. Immediate sensory and motor block were noted. The patient was then placed in the right lateral decubitus position for caudal epidural catheter placement. After sterile preparation, the caudal epidural space was accessed using an 18-gauge Tuohy needle. A 20-gauge epidural catheter was advanced $5 \mathrm{~cm}$ into the epidural space to allow for a T10-12 blockade. The needle was removed and the catheter secured. A test dose of lidocaine 1.5\% with epinephrine 1:200,000 (1 $\mathrm{mL}$ ) was negative. For the first $40 \mathrm{~min}$ of the procedure, the patient slept comfortably without need for additional medications. After $40 \mathrm{~min}$, dexmedetomidine $(8 \mu \mathrm{g})$ was administered intravenously for irritability with good effect. At $1 \mathrm{~h}$ post-incision, $0.2 \%$ ropivacaine $(6 \mathrm{~mL})$ was administered via the caudal epidural catheter and another dose of dexmedetomidine $(4 \mu \mathrm{g})$ was administered intravenously. The procedure, which lasted approximately $2 \mathrm{~h}$, was completed successfully. The patient was hemodynamically stable and required no supplemental oxygen throughout the operation. The caudal epidural catheter was removed in the operating room following completion of the surgery. Her PACU stay was uncomplicated, and she was discharged to home the next morning without incident.

\section{Discussion}

Our institution has developed a SA program to offer an alternative to GA for appropriate cases in light of concerns related 
to potential anesthetic neurotoxicity. Although the use of continuous SA, continuous caudal anesthesia, and SA combined with epidural anesthesia have been previously described (Table $1,[5-9])$, the majority of these reports have used the technique for avoidance of GA in a high-risk child [5-10]. Our goal with the reported technique (SA plus placement of a caudal epidural catheter) was to develop a technique that could be offered as a viable alternative to GA for longer surgical procedures, especially given recent concerns of the potential long term neurocognitive effects of general anesthetic agents. The spinal plus caudal epidural catheter technique can be used to circumvent the temporal limitations of SA, and in theory can be used for any length surgical procedure. Herein we report our successful experience with the technique in two infants to illustrate the technical aspects of the procedure as well as options for medication dosing.

Although SA in children is a relatively simple, elegant technique, it is not without limitations. As it is a single-shot technique, it can only be used for procedures that last approximately 1 hour $[11,12]$. Adjunctive agents such as epinephrine or clonidine can be added to the local anesthetic agent that may extend that duration $20-30 \%$, but the upper limit of duration remains 70 - 75 minutes [13]. Additionally, SA is generally reserved for pelvic and lower abdominal procedures. With this combined technique, it may be possible to perform more cephalad surgeries because a higher level of surgical anesthesia is possible with more cephalad placement of the tip of the epidural catheter.

In addition to avoiding potential neurotoxicity, SA provides several advantages over GA. SA provides superior intraoperative hemodynamic stability (decreased hypotension and bradycardia) when compared to GA [14]. Additionally, SA has been shown to lead to more effective blunting of the surgical stress response, lack of a need for airway management or supplemental oxygen, and limited need for parenteral opioids [10]. While SA has typically been limited to procedures lasting less than 70 - 75 minutes, this report presents an effective technique combining spinal and caudal epidural anesthesia for two prolonged urologic procedures lasting more than $90 \mathrm{~min}$. Combining methods of regional anesthesia can provide a surgical team with an extended window of time to perform surgical procedures. Furthermore, the surgical anesthesia provided by the initial SA provides effective anesthesia for placement of the caudal epidural catheter without the need for additional agents. Given the prolonged duration of the procedure, as noted in both of our patients, supplemental sedation may be required during the procedure given the cognitive level and limited ability of infants and children to remain quiet for a 2-h surgical procedure. Our experience with the technique and its efficacy have allowed us to expand its application outside of the neonatal and infant age range as our patients were 2 years and 16 months of age, respectively. Key to the success of this regional instead of general anesthesia program has been the cooperation of a well-trained, multidisciplinary team of surgeons, pediatric anesthesiologists, and nurses.

\section{Conflict of Interest}

None.

\section{Financial Support}

None.

\section{Author Contributions}

All authors contributed equally to this manuscript.

\section{References}

1. Welborn LG, Rice LJ, Hannallah RS, Broadman LM, Ruttimann UE, Fink R. Postoperative apnea in former preterm infants: prospective comparison of spinal and general anesthesia. Anesthesiology. 1990;72(5):838-842.

2. William JM, Stoddart PA, Williams SA, Wolf AR. Postoperative recovery after inguinal herniotomy in ex-premature infants: comparison between sevoflurane and spinal anaesthesia. Br J Anaesth. 2001;86(3):366-371.

3. Davidson AJ. Anesthesia and neurotoxicity to the developing brain: the clinical relevance. Paediatr Anaesth. 2011;21(7):716-721.

4. Blaylock M, Engelhardt T, Bissonnette B. Fundamentals of neuronal apoptosis relevant to pediatric anesthesia. Paediatr Anaesth. 2010;20(5):383-395.

5. Williams RK, McBride WJ, Abajian JC. Combined spinal and epidural anaesthesia for major abdominal surgery in infants. Can J Anaesth. 1997;44(5 Pt 1):511-514.

6. Somri M, Tome R, Yanovski B, Asfandiarov E, Carmi N, Mogilner J, David B, et al. Combined spinal-epidural anesthesia in major abdominal surgery in high-risk neonates and infants. Paediatr Anaesth. 2007;17(11):10591065.

7. Arora MK, Nagaraj G, Lakhe ST. Combined spinal-epidural anesthesia for a child with Freeman-Sheldon syndrome with difficult airway. Anesth Analg. 2006;103(6):1624.

8. Tobias JD. Anesthetic care for the child with Morquio syndrome: general versus regional anesthesia. J Clin Anesth. 1999;11(3):242-246.

9. Tobias JD, Lowe S, O'Dell N, Pietsch JB, Neblett WW, 3rd. Continuous regional anaesthesia in infants. Can J Anaesth. 1993;40(11):1065-1068.

10. Goeller JK, Bhalla T, Tobias JD. Combined use of neuraxial and general anesthesia during major abdominal procedures in neonates and infants. Paediatr Anaesth. 2014;24(6):553-560.

11. Frawley G, Bell G, Disma N, Withington DE, de Graaff JC, Morton NS, McCann ME, et al. Predictors of failure of awake regional anesthesia for neonatal hernia repair: data from the general anesthesia compared to spinal anesthesia study - comparing apnea and neurodevelopmental outcomes. Anesthesiology. 2015;123(1):55-65.

12. Williams RK, Adams DC, Aladjem EV, Kreutz JM, Sartorelli KH, Vane DW, Abajian JC. The safety and efficacy of spinal anesthesia for surgery in infants: the Vermont Infant Spinal Registry. Anesth Analg. 2006;102(1):67-71.

13. Rochette A, Raux O, Troncin R, Dadure C, Verdier R, 
Capdevila X. Clonidine prolongs spinal anesthesia in newborns: a prospective dose-ranging study. Anesth Analg. 2004;98(1):56-59, table of contents.

14. Ing C, Sun LS, Friend AF, Kim M, Berman MF, Paga- nelli W, Li G, et al. Differences in intraoperative hemodynamics between spinal and general anesthesia in infants undergoing pyloromyotomy. Paediatr Anaesth. 2017;27(7):733-741. 